\title{
Evaluation of fully automated motion corrected first pass myocardial perfusion MRI with semi quantitative perfusion parameter maps in patients with ischemic heart disease
}

\author{
Aya Kino ${ }^{1 *}$, Christopher Glielmi², Andrada R Popescu ${ }^{1}$, Mauricio S Galizia ${ }^{1}$, Jeremy Collins ${ }^{1}$, Darshit Thakrar ${ }^{1}$, \\ Jacob Fluckiger ${ }^{1}$, Hui Xue ${ }^{3}$, Jens Guehring ${ }^{3}$, Sven Zuehlsdorff², Daniel C Lee ${ }^{4}$, James Carr ${ }^{1}$
}

From 15th Annual SCMR Scientific Sessions

Orlando, FL, USA. 2-5 February 2012

\section{Summary}

The purpose of the study is to evaluate a fully automated motion corrected first pass myocardial perfusion (FPMP) MRI with semi quantitative perfusion parameter maps in patients with suspected ischemic heart disease.

\section{Background}

Coronary heart disease is the leading cause of death and disability in the US. FPMP MRI is increasingly used to assess ischemic heart disease; however respiratory motion is one of the major problems for myocardial blood flow quantification. An algorithm for motion correction, surface coil correction, temporal denoising and robust pixel-wise parameter map generation model was previously desribed [Xue H et al MICCAI 2009).This work evaluates automated workflow in a clinical setting to diagnose ischemic heart disease comparing free breathing and motion correcteted images and corresponding pixel-wise parameter map.

\section{Methods}

Stress and rest FPMP images were acquired using a 1.5T scanner (MAGNETOM Avanto, Siemens Healthcare) in 39 patients with suspected ischemic heart disease. Short axis slices were acquired during infusion of 0.075 $\mathrm{mMol} / \mathrm{kg}$ of Gadolinium (Magnevist, Bayer HealthCare Pharmaceuticals, USA) and adenosine (Adenoscan, AstellasPharma, USA) infusion $(0.14 \mathrm{mg} / \mathrm{kg} / \mathrm{min}$; duration: $4 \mathrm{~min}$ ) was administrated to induce stress. Free breathing, motion-corrected images and corresponding perfusion maps were assessed by 2 radiologists independently using the AHA 16 model and evaluated using a four point Likert scale (poor to excellent) to evaluate image quality and confidence level in presence or absence of hypo-perfusion regions. Upslope index of both free breathing and motion corrected images during stress and rest were manually calculated in nonischemic and ischemic areas and compared to the corresponding pixel-wise parameter map generated based on motion corrected images. FPMP MRI results were subsequently compared to coronary angiogram, stress echocardiography, or SPECT.

\section{Results}

Perfusion defects were detected in 25 patients (representative patient shown in Fig. 1). Mean image quality score for motion corrected images $(3.48 \pm 0.50)$ and confidence level $(3.31 \pm 0.41)$ were significantly higher $(\mathrm{p}<0.001)$ than free breathing images (mean image score of $2.51 \pm 0.63$ and confidence level of $2.83 \pm 0.58$ ). Upslope index of non ischemic and ischemic areas and semi quantitative perfusion parameter maps values were comparable $(P>0.05)$. Sensitivity and specificity for each technique, as well as the inclusion of perfusion parameter maps, are shown in Table 1. Although inline processed results showed higher sensitivity and specifity than standard images, differences were not significant $(\mathrm{p}<0.05)$.

${ }^{1}$ Radiology, Northwestern University, Chicago, IL, USA

Full list of author information is available at the end of the article

(c) 2012 Kino et al; licensee BioMed Central Ltd. This is an open access article distributed under the terms of the Creative Commons Attribution License (http://creativecommons.org/licenses/by/2.0), which permits unrestricted use, distribution, and reproduction in any medium, provided the original work is properly cited. 


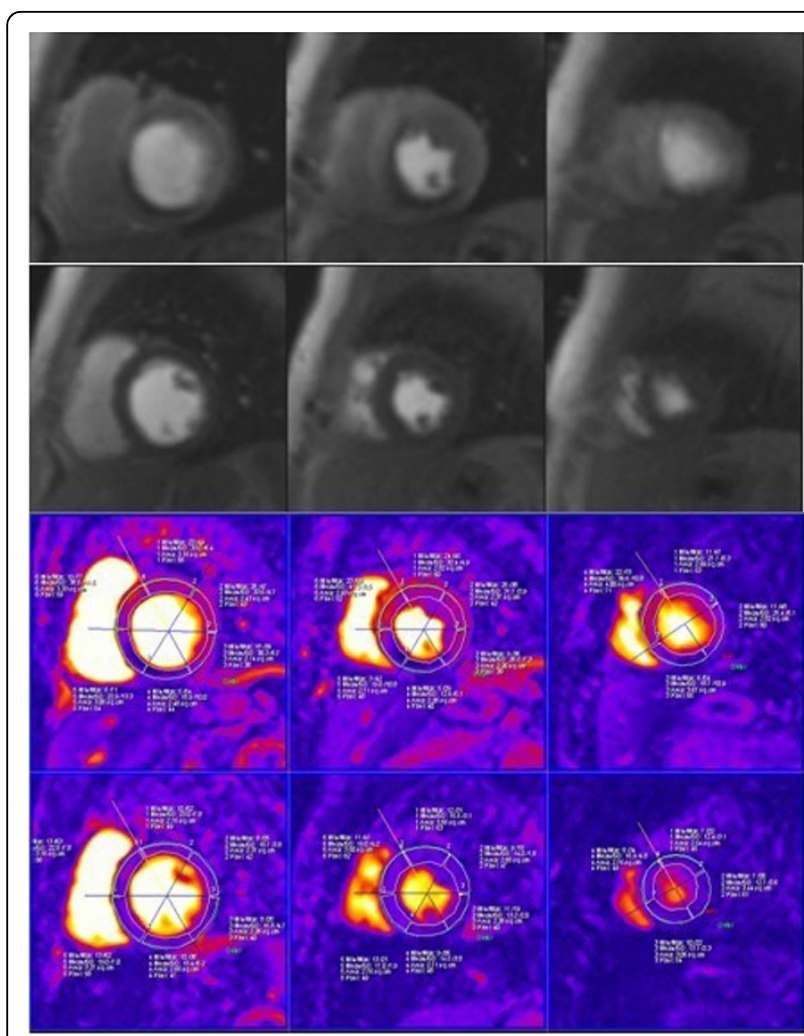

Figure 1 Perfusion images during the Stress (upper) and Rest (lower) and respective slope map from 57 years old woman with previous history of coronary artery disease post CABG with angina. Perfusion defects are seen at the basal mid and apical septal inferior and inferior regions corresponding to a RCA and LAD stenosis and map slopes values are not significant different during stress and rest images at the corresponding segments. Her coronary angiography examination showed $100 \%$ stenosis at LAD with LIMA graft patent, $70 \%$ in stent stenosis and $80 \%$ stenosis at RCA.

Table 1 Sensitivity and Specificity values from both readers by technique per subject.

\begin{tabular}{lccccccc}
\hline Reader 1 & FB & MC & MC + map & Reader 2 & FB & MC & MC + map \\
\hline Sensitivity & 0.85 & 0.89 & 0.89 & Sensitivity & 0.89 & 0.91 & 0.91 \\
Specificity & 0.82 & 0.85 & 0.85 & Specificity & 0.87 & 0.90 & 0.90 \\
\hline
\end{tabular}

Sensitivity and Specificity by reader and by technique. There is a increase in sensitivity and specificity during $M C$ images and $M C+$ map images.

\section{Conclusions}

A fully automated motion corrected first pass myocardial perfusion (FPMP) MRI with semi quantitative perfusion parameter maps showed comparable accuracy for detection of significant coronary artery disease in patients with ischemic disease.

\section{Funding}

Astellas Pharma Global Development, Inc.

\section{Author details}

${ }^{1}$ Radiology, Northwestern University, Chicago, IL, USA. ${ }^{2}$ Siemens Healthcare,

Chicago, IL, USA. ${ }^{3}$ Siemens Corporate Research USA, Princeton, NJ, USA.

${ }^{4}$ Dept Medicine-Division of Cardiology, Northwestern University, Chicago, IL, USA.

Published: 1 February 2012

doi:10.1186/1532-429X-14-S1-P249

Cite this article as: Kino et al:: Evaluation of fully automated motion corrected first pass myocardial perfusion MRI with semi quantitative perfusion parameter maps in patients with ischemic heart disease. Journal of Cardiovascular Magnetic Resonance 2012 14(Suppl 1):P249.

\section{Submit your next manuscript to BioMed Central} and take full advantage of:

- Convenient online submission

- Thorough peer review

- No space constraints or color figure charges

- Immediate publication on acceptance

- Inclusion in PubMed, CAS, Scopus and Google Scholar

- Research which is freely available for redistribution 